\title{
Sparse Hardware Embedding of Spiking Neuron Systems for Community Detection
}

\author{
KATHLEEN E. HAMILTON, NEENA IMAM, and TRAVIS S. HUMBLE, \\ Oak Ridge National Laboratory
}

\begin{abstract}
We study the applicability of spiking neural networks and neuromorphic hardware for solving general optimization problems without the use of adaptive training or learning algorithms. We leverage the dynamics of Hopfield networks and spin-glass systems to construct a fully connected spiking neural system to generate synchronous spike responses indicative of the underlying community structure in an undirected, unweighted graph. Mapping this fully connected system to current generation neuromorphic hardware is done by embedding sparse tree graphs to generate only the leading-order spiking dynamics. We demonstrate that for a chosen set of benchmark graphs, the spike responses generated on a current generation neuromorphic processor can improve the stability of graph partitions and non-overlapping communities can be identified even with the loss of higher-order spiking behavior if the graphs are sufficiently dense. For sparse graphs, the loss of higher-order spiking behavior improves the stability of certain graph partitions but does not retrieve the known community memberships.
\end{abstract}

CCS Concepts: • Mathematics of computing $\rightarrow$ Graph algorithms; $\bullet$ Hardware $\rightarrow$ Emerging architectures; Neural systems;

Additional Key Words and Phrases: Optimization, community detection, neural network, graph algorithm

\section{ACM Reference format:}

Kathleen E. Hamilton, Neena Imam, and Travis S. Humble. 2018. Sparse Hardware Embedding of Spiking Neuron Systems for Community Detection. J. Emerg. Technol. Comput. Syst. 14, 4, Article 40 (November 2018), 13 pages.

https://doi.org/10.1145/3223048

\section{INTRODUCTION}

IBM's TrueNorth Synaptic Processor offers a platform to execute deep learning tasks with high efficiency and low power consumption (Merolla et al. 2014) using event-based computation. This requires new algorithmic design and the adaptation of backpropagation (Esser et al. 2015) and

This work was supported by the United States Department of Defense and used resources of the Computational Research and Development Programs at Oak Ridge National Laboratory. This manuscript has been authored by UT-Battelle, LLC, under Contract No. DE-AC0500OR22725 with the U.S. Department of Energy. The United States Government retains and the publisher, by accepting the article for publication, acknowledges that the United States Government retains a nonexclusive, paid-up, irrevocable, world-wide license to publish or reproduce the published form of this manuscript, or allow others to do so, for the United States Government purposes. The Department of Energy will provide public access to these results of federally sponsored research in accordance with the DOE Public Access Plan.

Authors' addresses: K. E. Hamilton, N. Imam, and T. S. Humble, Oak Ridge National Laboratory, One Bethel Valley Road, Oak Ridge, TN 37831 USA; emails: \{hamiltonke, imamn, humblets\}@ornl.gov.

Permission to make digital or hard copies of all or part of this work for personal or classroom use is granted without fee provided that copies are not made or distributed for profit or commercial advantage and that copies bear this notice and the full citation on the first page. Copyrights for components of this work owned by others than ACM must be honored. Abstracting with credit is permitted. To copy otherwise, or republish, to post on servers or to redistribute to lists, requires prior specific permission and/or a fee. Request permissions from permissions@acm.org.

(C) 2018 Association for Computing Machinery.

1550-4832/2018/11-ART40 \$15.00

https://doi.org/10.1145/3223048 
Gibbs sampling (Das et al. 2015) have aided the deployment of convolutional neural networks (Esser et al. 2016) and restricted Boltzmann machines (Pedroni et al. 2016) on the TrueNorth system.

We have recently developed an approach to spiking neural network design that we applied to the graphical problem of community detection. This problem does not generate large sets of training data, and it may not be possible to extract features from one instance for use on a different instance. The problem of community detection can be cast as a graph partitioning problem, which Hopfield networks (Hopfield 1982) can be used to solve (Hertz et al. 1991). Hopfield networks are a class of recurrent neural networks that have been used to solve many kinds of optimization problems and pattern retrieval tasks (Hopfield and Tank 1985) but do not have any hidden variables. Additionally, the Hopfield network parameters may be set through heuristics (e.g., Hebbian learning rules) rather than an adaptive learning process.

This article describes a hardware implementation of research described in References (Hamilton et al. 2017; Hamilton and Humble 2018). We summarize our previous results thusly: In Hamilton et al. (2017), we established that a mapping can be made from an undirected graph to a fully connected spiking neural system that can be driven by external current to generate spiking responses that can identify individual communities using a Hamming distance-based metric; in Hamilton and Humble (2018), spiking neural systems can be incorporated into larger community detection workflows, such as label propagation. In these two previous works, we have relied on numerical simulation of spiking neuron dynamics to generate the spiking neuron responses.

In this article, we aim to execute the spiking label propagation algorithm from Hamilton and Humble (2018) using IBM's TrueNorth to generate the spiking neuron output. The spiking neuron networks embedded onto the neuromorphic processor to generate spiking output differs significantly from the spiking neuron systems we have previously studied with numerical simulation. We do not embed fully connected systems of spiking neurons on TrueNorth; instead, we embed disconnected subtrees of neurons extracted from the original graph. This article presents results that show how the sparsity of the embedding affects the ability to detect communities in random benchmark graphs.

Our approach to label propagation relies on a Hamming metric that has previously been used to identify similar spike trains (Humphries 2011). Incorporating spikes into a larger label propagation workflow is dependent on the linear separability of this Hamming metric. This article is organized as follows: We first review the dynamics of fully connected, spin-glass spiking neural systems. By highlighting the dominant spiking responses, we then show how these fully connected systems are sparsely mapped into a neuromorphic system with discrete dynamics. We determine how sparse embedding of an undirected graph onto current generation neuromorphic hardware affects the generated firing patterns and the linear separability of the Hamming metric. We then use the output generated on TrueNorth in the spiking label propagation algorithm designed in Hamilton and Humble (2018) and summarized in Algorithm 1.

\section{SPIN-GLASS SPIKING NEURAL NETWORK CONSTRUCTION}

\subsection{Definitions}

Our method identifies groups of strongly connected vertices in undirected, unweighted graphs $\mathcal{G}=\mathcal{G}(V, E)$. These graphs do not contain self-loops, multiple edges, or weighted edges and are fully defined using a vertex set $V(\mathcal{G})=\left\{v_{i}\right\}$ and a set of symmetric edges $\left(E(\mathcal{G})=\left\{e_{i j}\right\}, e_{i j}=e_{j i}=\right.$ $\left.\left(v_{i}, v_{j}\right)\right)$. In this article, we focus on identifying communities with respect to a set of known labels. The benchmark graphs used in this article (see Table 1) are generated with a fixed number of communities and each vertex is assigned membership to only one community.

In a previous article (Hamilton and Humble 2018), we defined these systems of spiking neurons as "spin-glass spiking neural networks" (SGSNNs) These are edge-weighted networks 
$\mathcal{S}=\mathcal{S}\left(N, W, s_{W}\right)$; using a neuron set $(N=\{n\})$, a set of symmetric connections $\left(W=\left\{w_{i j}\right\}\right.$, $\left.w_{i j}=w_{j i}=\left(n_{i}, n_{j}\right)\right)$ called synapses. Associated with each synapse is an edge weight $\left(s_{w}\right)$ that may be positive or negative, and self-connections $\left(w_{i i}=\left(n_{i}, n_{i}\right) \notin W\right)$ are not allowed. The spiking dynamics generated by the positive and negative synaptic connections is analogous to the dynamics of a spin-glass that can exhibit strong local correlations that do not propagate globally throughout the entire system. Likewise, in a SGSNN the spiking response to an external current can generate local spiking behavior without spreading to all neurons.

\subsection{Spiking Behavior}

For community detection, it is necessary to generate localized spiking output that can be attributed to a driven neuron. Specifically, we want to identify which neurons fire in response to an individual neuron firing at a uniform rate $\left(f_{0}\right)$. Our identification of a community assumes that neurons in the same community will exhibit similar spiking behavior as all neurons in a system are driven, individually. Similarly to the well-known phrase in Hebbian learning "neurons that fire together, wire together" (Lowel and Singer 1992), we build our community detection methods assuming "neurons wired together, fire together."

Using steplike external currents, we can generate uniform spiking from individual neurons. These spikes will have an excitatory effect on neurons connected by a positive synapse and an inhibitory effect on neurons connected by a negative synapse. Additionally, as positive weighted spikes arrive at a neuron, that neuron can be driven above its firing threshold, causing further spiking. For a SGSNN we identify four unique spike responses:

Driven spiking. $I(t)=I_{\text {ext }}$ is the constant external current applied to a neuron through a square pulse current. This causes a neuron to fire at a uniform rate $f_{0}$ that is dependent on the height of the square pulse, the time constant $\tau$ and the duration of the pulse $t_{A}$. This spiking response is shown in Figure 1(b).

Response spiking. $I(t)=\sum_{t_{f}} s_{w} \delta\left(t-t_{f}\right)$, spikes arriving at a neuron change the neuron's membrane potential by $\Delta V=s_{w}$. If a neuron is directly connected to a driven neuron, then $s_{w}>0$ and spikes arriving can eventually cause a neuron, not being driven by external current, to fire a spike. This response is shown in Figure 1(c).

Inhibited spiking. $I(t)=\sum_{t_{f}} s_{w} \delta\left(t-t_{f}\right)$, but if a neuron is not directly connected to a driven neuron, then $s_{w}<0$ and spikes arriving will depress the membrane potential of a neuron, preventing it from firing a spike. This response is shown in Figure 1(d).

Higher-order spiking. If multiple length-2 paths exist between a driven neuron and a nonadjacent neuron, then the arrival of response spikes can offset the potential depression caused by inhibitory driving spikes. This response is shown in Figure 1(e).

\section{NEUROMORPHIC EMBEDDING}

When mapping a SNN to IBM's TrueNorth, we minimize the number of neurons and synapses used and avoid embedding a fully connected spin-glass system. Returning to the spiking dynamics of a spin-glass spiking neuron system, we embed disconnected subtrees of the original graph that then are used to generate the driven and response spiking behavior described in Description 2.2. This is achieved using short, disconnected subtrees formed by a root vertex $v_{0}$ and its neighbors $\left\{v_{i}: v_{i} \in\right.$ $\left.V(\mathcal{G}), e_{i 0} \in E(\mathcal{G})\right\}$. Mapped to a set of neurons, the tree $\mathcal{T}\left(n_{0}\right)$ has $D+1$ neurons and $D$ synapses with the same (positive) weight $s_{w}=1$ (see Figure 2), where $D$ is the degree of the vertex $v_{0}$.

In Figure 3, the difference between two spike rasters generated for the same 50 vertex graph are shown. The sparse tree-based embedding is able to replicate the leading-order firing dynamics of the spin-glass SNN. In addition to the reduction of network density, there are a number of 
(a)

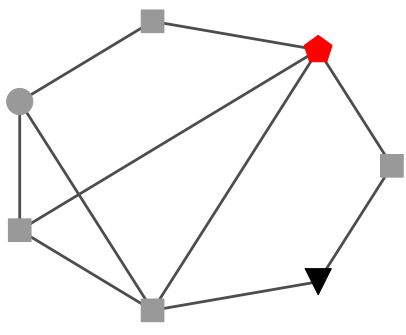

(b)

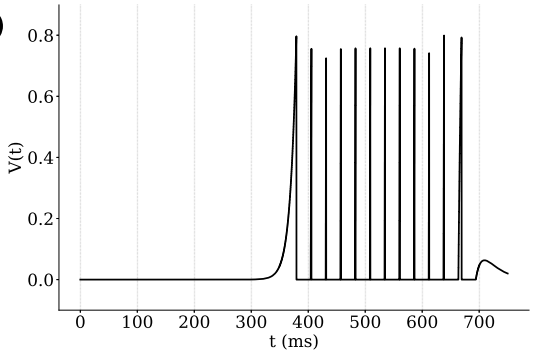

(c)

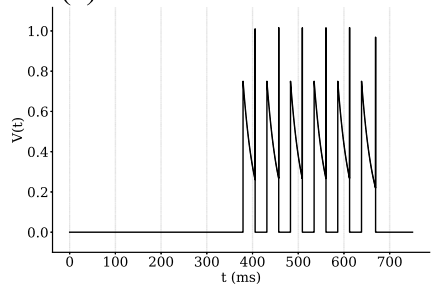

(d)

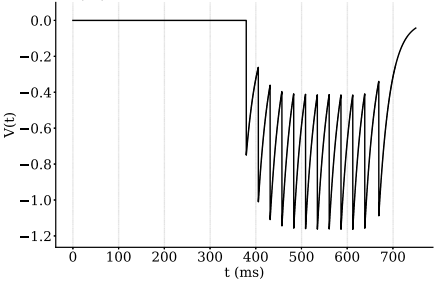

(e)

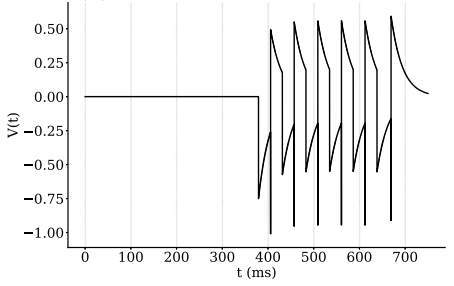

Fig. 1. The four classes of spiking behavior for simulated neuron dynamics of a fully connected SGSNN. A simple graph on seven vertices that is not fully connected is shown in (a). When mapped to a fully connected SNN (not shown), a square pulse external current is applied to a single neuron (red, pentagon) causing it to fire spikes at a fixed rate (b) and the remaining neurons of the graph exhibit one of three possible spike responses ((c)-(e)). The nearest neighbors (grey, square) will only receive positively weighted spikes and will eventually spike (c). A neuron that is disconnected from the driven neuron (black, triangle) and additionally has few length-2 paths connecting to the driven neuron will receive negatively weighted spikes from the driven neuron, slightly offset by positively weighted spikes arriving from any neighbors. However, the arrival of the positively weighted spikes are insufficient to cause this neuron to spike (d). A neuron that is disconnected from the driven neuron (grey, circle) but has many length-2 paths connecting it to the driven neuron will receive negative weighted spikes from the driven neuron, but the positive weighted spikes from nearest-neighbor neurons can strongly offset the inhibitory effects and this neuron will eventually spike (e).

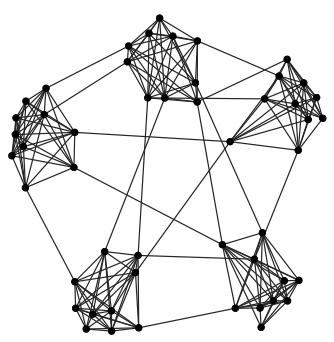

(a)

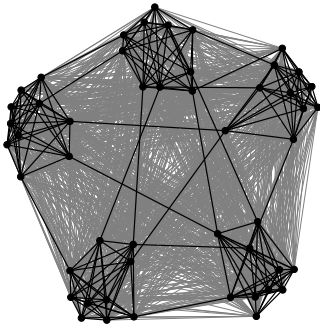

(b)

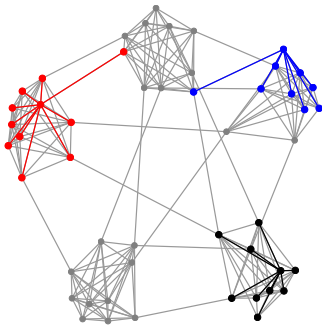

(c)

Fig. 2. Two embeddings of a relaxed caveman graph on 50 vertices: (a) The relaxed caveman graph on 50 vertices. (b) The graph embedded into a fully connected, 50-neuron SNN with 225 excitatory synapses (black) and 1,000 inhibitory synapses (grey). (c) The embedding onto the TrueNorth system using a set of disconnected subtrees (red, blue, black), each with $D_{i}+1$ neurons and $D_{i}$ excitatory synapses determined by the degree of each graph vertex. 


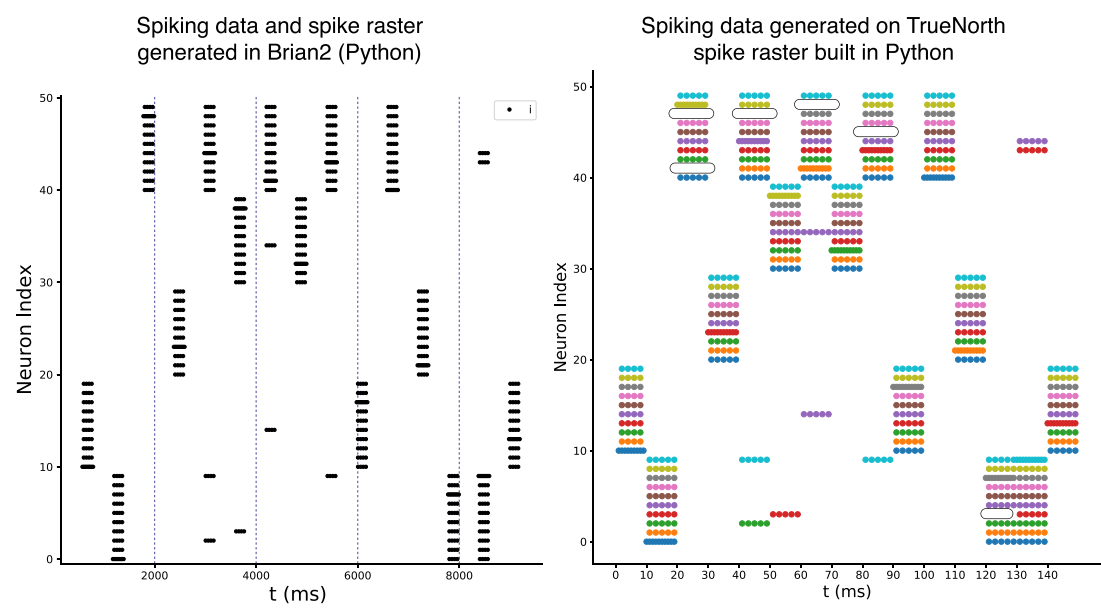

Fig. 3. Spike rasters for a relaxed caveman graph on 50 vertices. (Left) The spike raster generated with a fully connected, spin-glass system and simulating the spiking neuron equations of motion using the Python library Brian2. (Right) The spike raster built from TrueNorth spiking output generated using the sparse, tree embedding. The ovals highlight where neurons do not fire due to the lack of support for higher-order spike responses.

simplifications that are possible using the tree-based embedding. The lack of any higher-order spiking behavior, or inhibitory spiking behavior also allows for a simpler neuron model. Each leaky integrate and fire neuron is defined only by its firing threshold $v_{t h}$. In contrast to the SGSNN construction, the sparse hardware embedding using heterogeneous parameters. Each subtree is embedded as a chain of tonic spiking neurons (Cassidy et al. 2013). The root of the subtree has a unit firing threshold $\alpha_{0}=1$ so that any spike it receives it immediately fires. All neighboring vertices have a firing threshold $\alpha_{i}=2$ so that two spikes must arrive from the root of the subtree before any of the remaining neurons fire.

Spiking output is generated in the SGSNN construction by applying a steplike external current to a single neuron and recording the output of all neurons that spike (including the driven neuron). To clearly distinguish that neurons are firing in response to a specific driven neuron, the time gap between subsequent pulses has to be long enough to allow any incurred potential changes to decay away. On TrueNorth, spiking output is generated by feeding a discrete set of spikes (spaced at $1 \mathrm{~ms}$ intervals) to the root neuron of an embedded subtree, and recording the spiking output of all neurons in the subtree (including the root neuron). The output generated on TrueNorth is made by successive calls to the hardware, with each call embedding a single subtree and recording all spike output. Each call is independent and it is not possible for the spiking output from tree $\mathcal{T}\left(n_{i}\right)$ to affect the spiking output of tree $\mathcal{T}\left(n_{i+1}\right)$. Thus the spiking output from TrueNorth can be generated with a much shorter gap between each effective "driven" neuron.

\section{IDENTIFYING KNOWN COMMUNITIES}

We measure the performance of spiking label propagation using a set of benchmark graphs each 128 vertices and known communities (see Table 1). We compare the results from TrueNorth to a set of numerical simulations of SGSNN systems. Using the Python library Brian2 (Goodman and Brette 2008), the numerical simulations constructed fully connected spiking neurons systems and solved for the spiking dynamics by integrating the system of equations for leaky-integrate and fire 
Table 1. Community Distribution for Benchmark Graphs Generated on 128 Vertices with Mean Degree $\langle D\rangle$

\begin{tabular}{lccc} 
Graph & $|E(\mathcal{G})|$ & $\left\{Q_{i}\right\}$ & $\langle D\rangle$ \\
\hline $\mathcal{G}_{A}$ & 256 & $(\{8\},\{(8,16)\})$ & 4 \\
\hline $\mathcal{G}_{B}$ & 512 & $(\{16\},\{(16,8)\})$ & 8 \\
\hline $\mathcal{G}_{C}$ & 768 & $(\{24,32\},\{(24,4),(32,1)\})$ & 12 \\
\hline $\mathcal{G}_{D}$ & 1024 & $(\{32\},\{(32,4)\}$ & 16 \\
\hline
\end{tabular}

neurons:

$$
\begin{gathered}
\frac{d V_{j}}{d t}=\frac{\left(V_{\text {ext }}(t)-V_{j}(t)\right)}{\tau}, \\
V_{\text {ext }}(t)=I_{\text {ext }}(t) R+\sum_{\substack{i \rightarrow j, i \neq j}} w_{i j} \delta\left(t-t^{(f)}\right) .
\end{gathered}
$$

The systems were constructed with the following neuron parameters: $v_{t h}=0.8 \mathrm{~V}, v_{0}=v_{R}=0.0 \mathrm{~V}$, $\tau=25 \mathrm{~ms}, t_{R}=20 \mathrm{~ms}$, and synaptic weight amplitude $\left|s_{w}\right|=0.75 \mathrm{~V}$. In Equation (2) a unit resistance term $R=1 \Omega$ is included.

The steplike pulse sequence consists of 128 homogeneous steplike pulses with shape parameters: $\beta=50.0 \mathrm{~s}^{-1}, A_{M A X}=12.0 \mathrm{~V}, t_{\text {pulse }}=200 \mathrm{~ms}$, and each pulse start was separated by $400 \mathrm{~ms}$, resulting in a gap between subsequent pulses of $200 \mathrm{~ms}$. During steplike pulse driving a neuron would fire a total of 10 spikes with frequency $\delta_{1} \approx 0.21 \mathrm{~ms}$. In response, its nearest neighbors will fire a total of $10 / 2=5$ spikes with frequency $\delta_{2} \approx 0.42 \mathrm{~ms}$.

The numerical simulations used low-order integration methods that led to slight variations in the firing rate, but the total number of spikes fired was not affected. Analogous to randomizing the vertex set in the algorithm of Raghavan et al. (2007) we randomize the order in which neurons are driven. On TrueNorth, each embedded subtree was given an input of $n$ spikes, with a gap of $1 \mathrm{~ms}$ between each subtree output. The root neuron outputs all $n$ input spikes, and any neighboring neuron will output $\lfloor n / 2\rfloor$ spikes. The neurons are also driven in a random sequence; however, the sequence used for the TrueNorth experiments was not the same as the sequence used to generate the Brian2 output.

Once the spiking output is generated (either through numerical simulation or on TrueNorth), the spike trains are decoded into binary vectors. Decoding a spike train $s_{i}$ is done by fixing a time window width $\Delta t$, and constructing a $L$-length vector $x_{i}$ where the $k$ th entry $x_{i}^{[k]}=1$ if a spike occurs within the time interval $\left[t_{k}, t_{k}+\Delta t\right]$, or $x_{i}^{[k]}=0$ if no spike occurs. The size of the time window determines the length of the final vector, $L=T / \Delta t$.

\section{SPIKING LABEL PROPAGATION}

The label propagation algorithm (Raghavan et al. 2007) assigns a label to a graph vertex that agrees with the majority of neighboring vertices' labels. In spiking label propagation, the label of a randomly chosen source neuron is assigned to all neurons that have similar spike trains (see Algorithm 1). The efficacy of the spiking label propagation is dependent on the linear separability of the Hamming metric,

$$
H\left(x_{i}, x_{j} ; \Delta t\right)=\left(1-\frac{h\left(x_{i}, x_{j}\right)}{L}\right)
$$

and it quantifies the similarity between two spike trains after they have been decoded into binary vectors. 


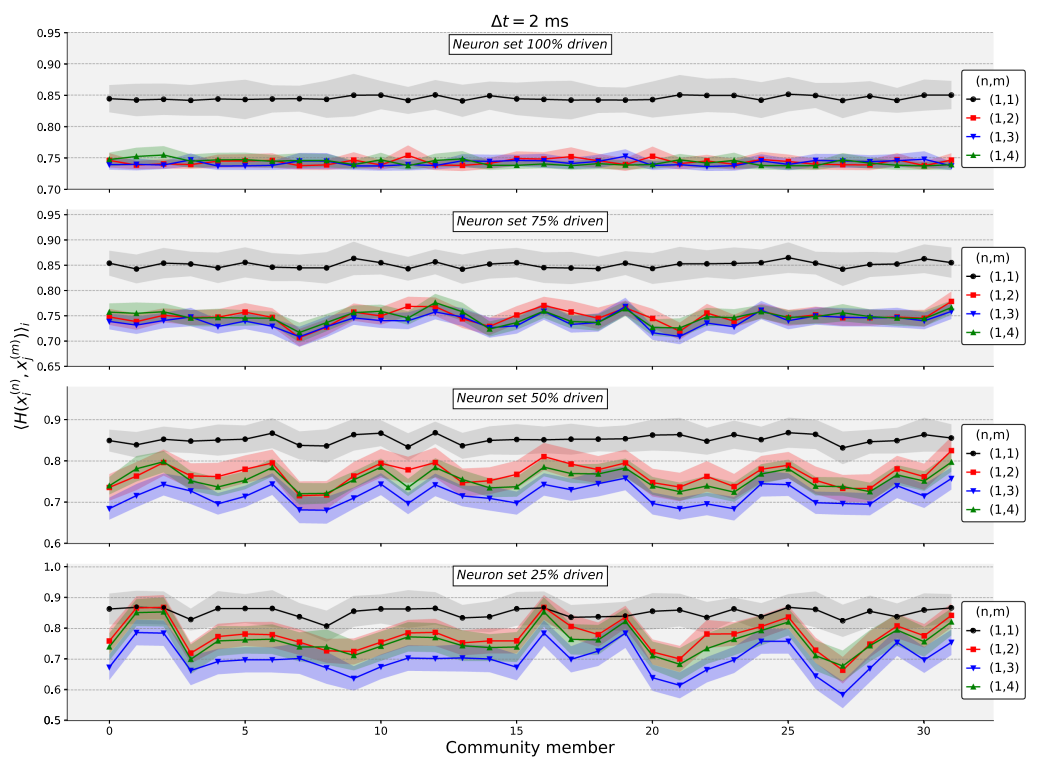

Fig. 4. (Color online) The mean Hamming metric $\left\langle H\left(x_{i}^{n}, x_{j}^{m}\right)\right\rangle_{i}$ for graph $\mathcal{G}_{D}$, averaged over all sources in Community 1 . The shaded regions cover $\langle H\rangle \pm \sigma(\langle H\rangle)$. The time step used to decode the spike trains was $\Delta t=2 \mathrm{~ms}$. Similar behavior is observed for Communities 2, 3, and 4 .

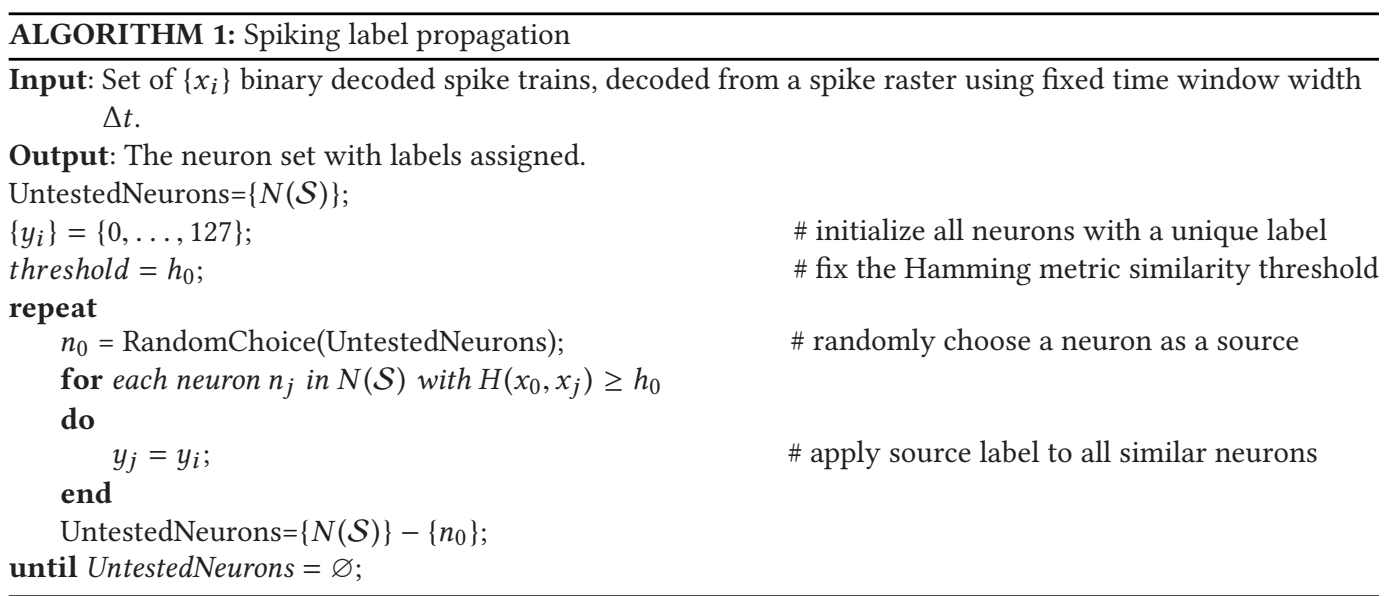

Spiking label propagation is most effective when the Hamming distance metric can be used to identify individual communities from the remainder of the graph. In Figures 4 and 5 the mean Hamming distance measure is plotted from spikes generated on TrueNorth, for graph instance $\mathcal{G}_{D}$ and any neuron chosen in Community 1 . A time window of width $\Delta t=2 \mathrm{~ms}$ is large enough to contain two spikes fired by the root neuron and one spike fired from neighboring neurons. This maximizes the similarity between all neurons in the embedded subtree. The Hamming metric measured between any two neurons in Community 1 is linearly separable from the Hamming metric measured between any neuron in Community 1 to any neuron in Communities 2,3, or 4 .

We generated spikes on TrueNorth by inputting a set of nine spikes to each root neuron, and there is a gap of $1 \mathrm{~ms}$ between the responses from separate trees. Thus, all spike responses that can 


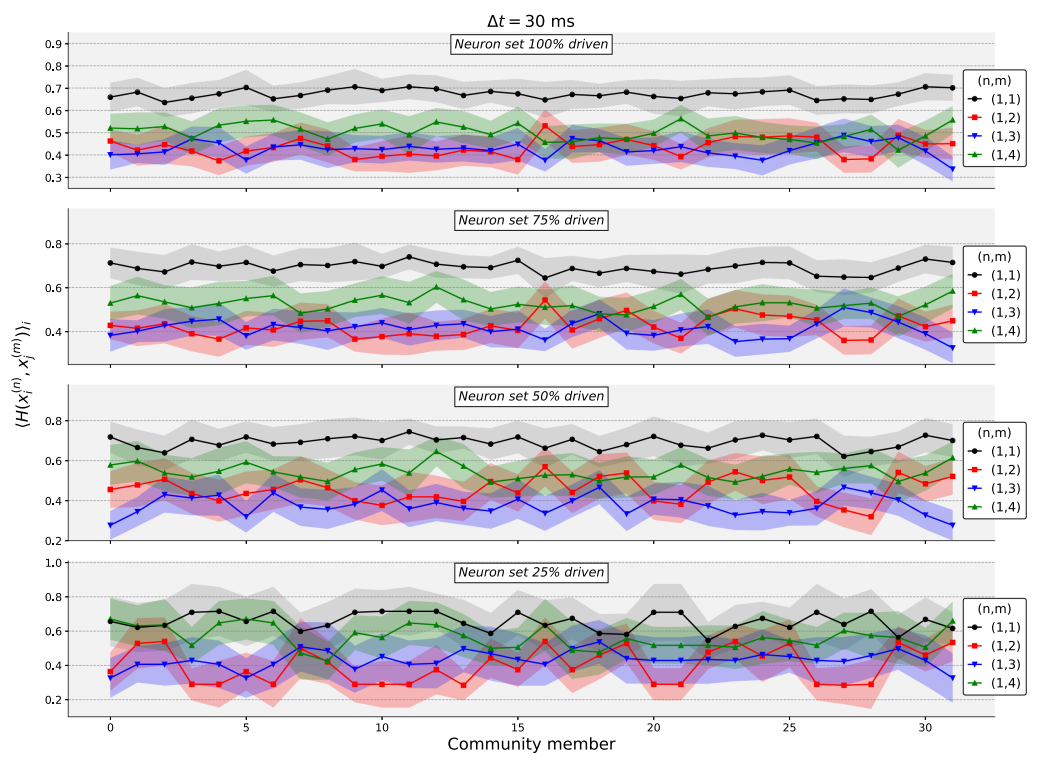

Fig. 5. (Color online) The mean Hamming metric $\left\langle H\left(x_{i}^{n}, x_{j}^{m}\right)\right\rangle_{i}$ for graph $\mathcal{G}_{D}$, averaged over all sources in Community 1 . The shaded regions cover $\langle H\rangle \pm \sigma(\langle H\rangle)$. The time step used to decode the spike trains was $\Delta t=30 \mathrm{~ms}$. Similar behavior is observed for Communities 2,3 , and 4 .

be attributed to a single root neuron will be contained within a time window of $\Delta t=10 \mathrm{~ms}$. If the time window is increased above this size, then the responses from multiple subtrees are decoded into identical binary vectors. A time window of width $\Delta t=30 \mathrm{~ms}$ results in binary vectors that cannot reliably distinguish individual communities.

Once the spiking output is generated on TrueNorth, its spike trains are decoded and passed into the label propagation algorithm. Each graph vertex is initialized with a unique label $\left\{y_{i}^{(I)}\right\}=$ $\{0 \ldots 127\}$. This set converges to a final set of labels $\left\{y^{(F)}\right\}$ that identify communities according to the Hamming metric. In Figure 6, the number of unique labels in the final neuron label set is plotted as a function of the Hamming metric $h_{0}$. All graphs have two trivial solutions: $\left\{y_{i}^{(F)}\right\}=\left\{y^{\prime}\right\}$ or $\left\{y_{i}^{(F)}\right\}=\{0 \ldots 127\}$. If the Hamming metric is fixed at a low value (e.g., $\left.h_{0} \approx 0.75\right)$, then all neurons are considered similar and the label set converges to a single label $y^{\prime}$. However, if the Hamming metric is fixed at a high value (e.g., $h_{0} \approx 0.99$ ), then no two neurons are similar and the label set does not diverge from the original values, and each neuron remains with a unique label (see Figure 7).

Depending on the connectivity of the graph, a range of $h_{0}$ values may exist for which $\left\{y^{(I)}\right\}$ will converge to a non-trivial set of labels. We have observed this for the benchmark graphs $\mathcal{G}_{B}, \mathcal{G}_{C}$, and $\mathcal{G}_{D}$ : values of $h_{0}$ that result in a final label set with $Q$ unique labels, where $Q$ is the known number of communities (see Figure 8). In addition to finding the correct number of communities, these labels correctly return the known community memberships (see Figure 9). We use the variance of information (Meilă 2007) to measure similarities between label sets. The variance of information between two equivalent label sets is zero.

\section{DISCUSSION}

The spike output generated on TrueNorth improves the stability of non-trivial solutions, seen in the increase in the range of $h_{0}$ values over which $\left|\left\{y_{i}^{(F)}\right\}\right|=Q$ (which we refer to as a "plateau"). In Hamilton and Humble (2018), we noted that the stability of a non-trivial solution could be 


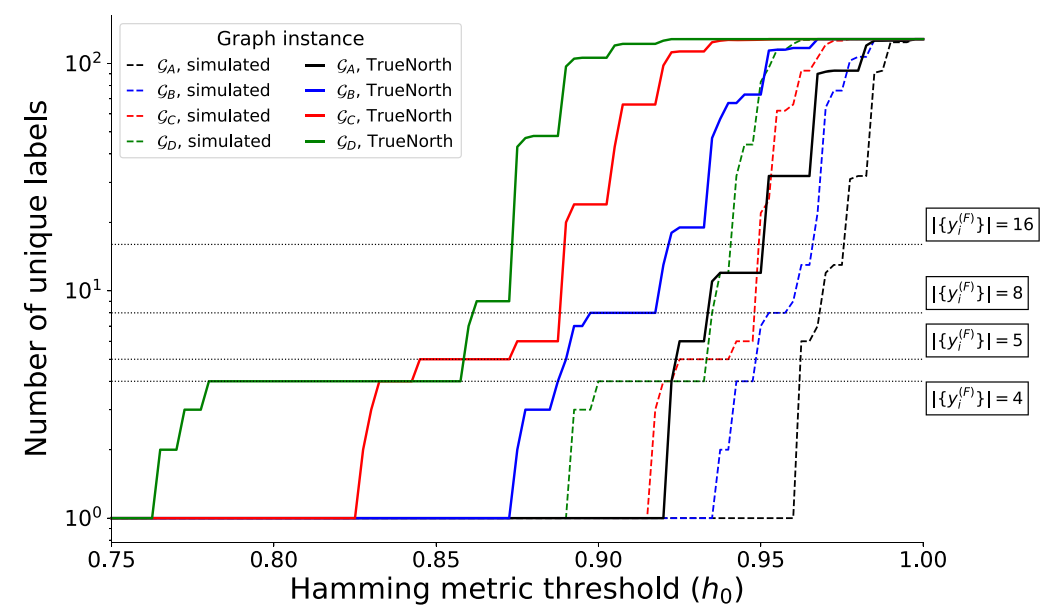

Fig. 6. (Color online) The number of unique labels in the final label set for graph instances $\mathcal{G}_{A}, \mathcal{G}_{B}, \mathcal{G}_{C}$, and $\mathcal{G}_{D}$. The solid lines show the final results for label propagation using spikes generated with the sparse tree embedding. The dashed lines show the final results for label propagation using spikes generated by integrating the equations of motion for the fully connected spin-glass system. Additional simulation parameters are given in the text.

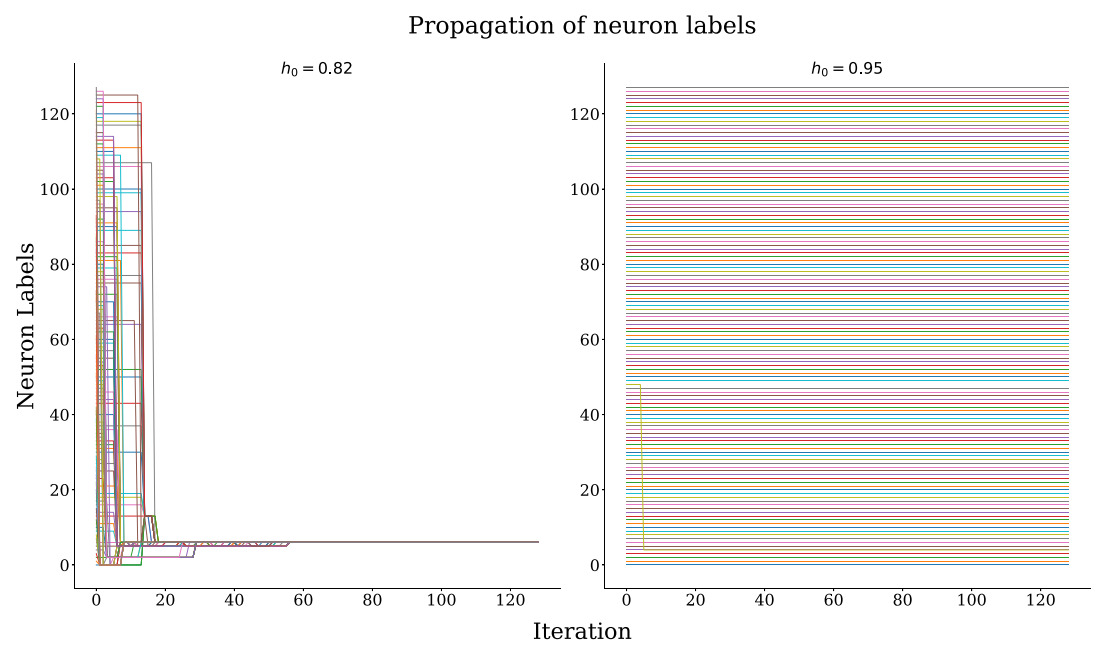

Fig. 7. (Color online) The convergence of a label set to trivial solutions for graph instance $G_{C}$, and spikes generated on TrueNorth. If the Hamming metric threshold is set too low $\left(h_{0}=0.82\right)$, then the initial set of 128 labels converges to a single label and all vertices are contained in the same community. If the Hamming metric threshold is set too high $\left(h_{0}=0.95\right)$, then few labels propagate across the system and most graph communities would just contain a single vertex.

improved by increasing the width of the driving pulse, causing a neuron to fire more spikes. After binary decoding, the resulting vectors $\left\{x_{i}\right\}$ will have a higher number of non-zero entries, which increases the Hamming distance between dissimilar spike trains. In Figure 6, the size of the final label sets for 4 graph instances, using spikes generated by numerical simulation or by generation on TrueNorth. Both systems generate comparable number of spikes when a neuron is driven (or 


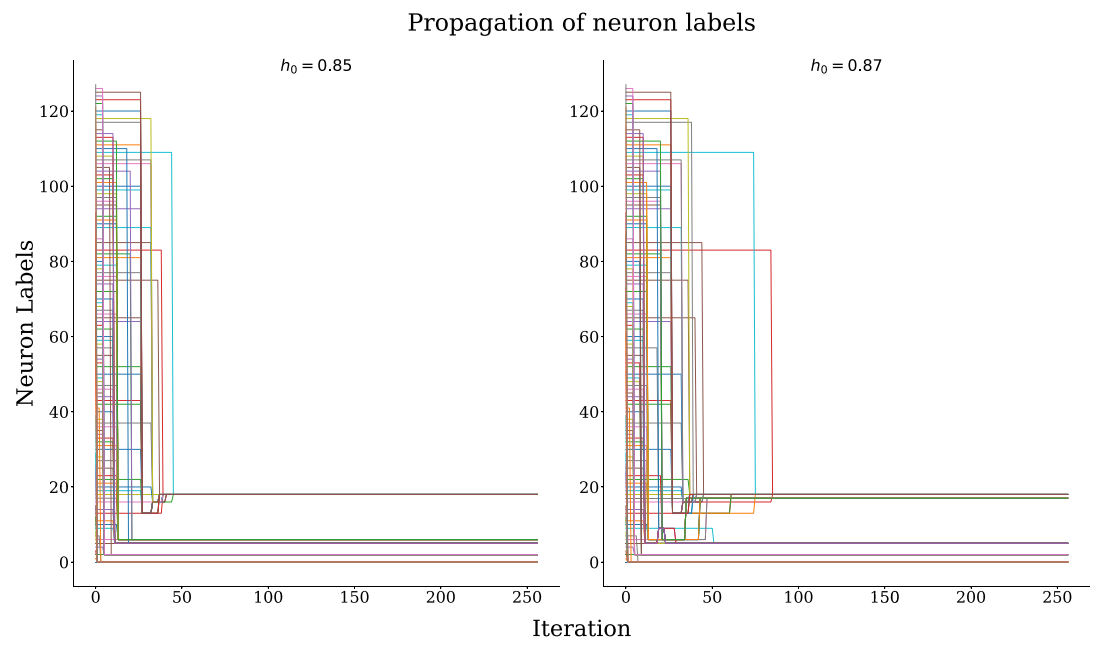

Fig. 8. (Color online) The convergence of a label set to non-trivial solutions for graph instance $\mathcal{G}_{C}$, and spikes generated on TrueNorth. There may exist a range of Hamming metric threshold values $\left(h_{0}=0.85\right.$ or $\left.h_{0}=0.87\right)$ where the initial set of 128 labels converges to a non-trivial set of final labels.

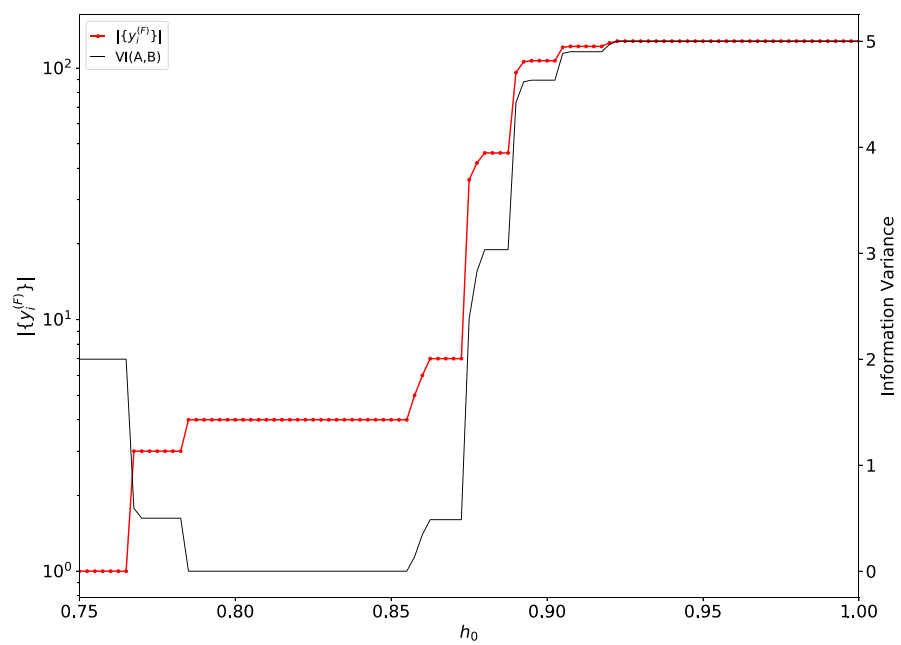

Fig. 9. (Color online) For graph instance $\mathcal{G}_{D}$ and using spikes generated on TrueNorth, there is a range of values for $h_{0}$ where the initial set of 128 labels converges to 4 unique labels (red). These communities match the known communities, demonstrated by the vanishing of the variance of information (black).

for TrueNorth, reads in a set of spikes). The pulse width $t_{A}=200 \mathrm{~ms}$ causes driven neurons to fire 10 spikes, and on TrueNorth each root neuron reads in a set of 9 spikes.

To generate Figure 10 we ran several numerical simulations with increasing values of $t_{A}$, while keeping the gap between pulses fixed at $200 \mathrm{~ms}$. For each pulse width a full spike raster was generated and decoded and then label propagation was run on the decoded output. The width of the plateau corresponds to the difference between the maximum and minimum values of $h_{0}$ for which the label set converged to a non-trivial set equal to the known number of communities. 


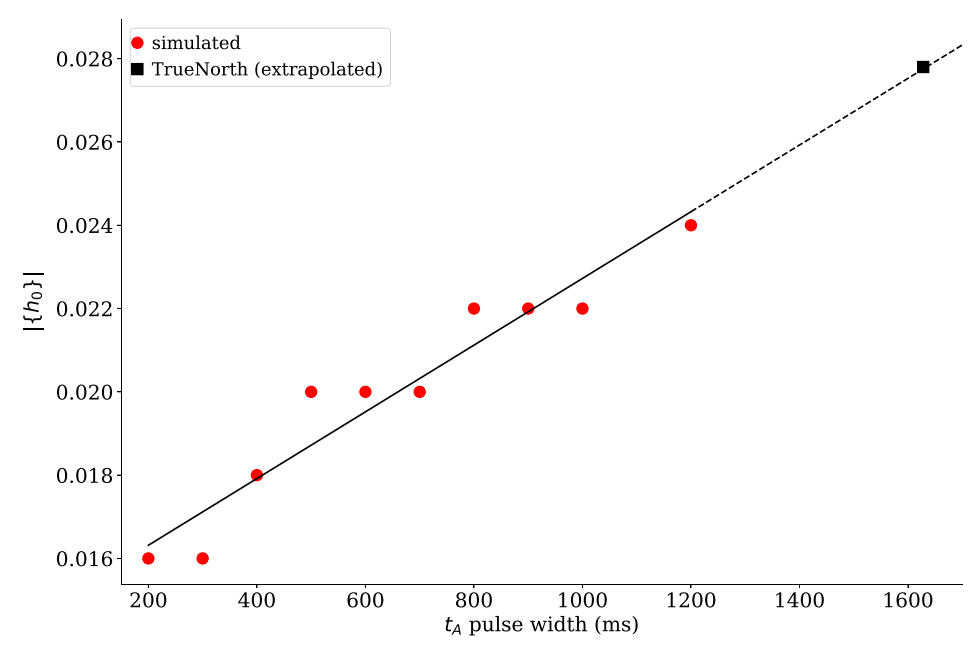

Fig. 10. The width of the plateau at $\left|\left\{y^{(F)}\right\}\right|=5$ for graph instance $\mathcal{G}_{C}$. Increasing the pulse width $t_{A}$ increases the plateau width for SGSNN systems (red, circles). We place the plateau width from the TrueNorth results (black, square) on an extrapolated linear fit line. To generate a comprable plateau width with numerical simulation would require a pulse width of $1,628 \mathrm{~ms}$.

This increased the number of nonzero entries in the resulting binary vectors, while keeping the number of zero entries constant. By increasing the nonzero entries in the decoded spike trains, we can increase the Hamming distance between dissimilar spike responses. Alternatively, the Hamming distance between similar spike trains could be minimized by varying the time window $\Delta t$ or using an alternative decoding method. In Humphries (2011), it was shown that using a "binless" decoding of spike trains rather than time discretized decoding led to a more robust community identification in noisy spike trains.

We compared the convergence behavior between the numerical simulations and the TrueNorth results based on a simple linear fit of the plateau widths as a function of driving pulse width. We extrapolate that it would be possible to generate similar convergence behavior and non-trivial solution stability between numerical simulation and the results generated using TrueNorth (see Figure 10) if the pulse width was increased to $t_{A}=1628 \mathrm{~ms}$. In contrast, the results from TrueNorth could be obtained from an effectively driven neuron firing only 9 spikes to its neighbors, while comparable results obtained through numerical simulation would require a driven neuron to fire approximately $\lceil 1628 / 21\rceil=78$ spikes.

For the benchmark graph set, the spiking label propagation method was able to return the known community memberships and improved the stability of non-trivial solutions, except for graph instance $\mathcal{G}_{A}$. For this graph instance, neither the simulated system or the system deployed on TrueNorth converged to a non-trivial label set of size $\left|\left\{y_{i}^{(F)}\right\}\right|=16$, though the TrueNorth results showed an increase in the plateau width at $\left|\left\{y_{i}^{(F)}\right\}\right|=12$. Graph instance $\mathcal{G}_{A}$ is unique in that the spike response generated by a fully connected SNN is the closest to the spike response generated by the TrueNorth embedding. On $\mathcal{G}_{A}$ multiple length-2 paths between vertices are not as prevalent as on the other benchmark graphs, and there are few neurons that generate the higherorder spiking behavior shown in Figure 1(e).

We believe this underperformance is correlated to the small size of the spike response and emphasizes the necessity of higher-order spike responses for identifying communities. While determining the optimal size of the localized spike response is still an open question, our label 
propagation method is dependent on localized spike responses that significantly overlap for neurons in the same community.

To further increase the size of spike responses, it will be necessary to embed larger subgraphs into TrueNorth. While some inhibitory synapses will need to be used, the embedding should not be fully connected. For sparse graphs or communities mapped a fully connected spiking neuron systems, it is possible that a high density of inhibitory synapses will overly restrict the spike response and lead to spike responses that do not significantly overlap for neurons in the same community. Future work is needed to determine the optimal size of a subgraph to embed onto TrueNorth.

\section{CONCLUSIONS}

This article is the third in a series of articles that detail how a graphical problem (community detection) can be solved using spiking neurons and neuromorphic systems. In previous articles (Hamilton et al. 2017; Hamilton and Humble 2018), we focused on three areas: how an undirected graph can be mapped to a fully connected system of spiking neurons; how a characteristic set of spiking outputs can be generated; and, finally, how these spiking outputs can be incorporated into label propagation. In this article, we have discussed the same three areas with respect to deployment on IBM's TrueNorth processor. By focusing on replicating leading-order spiking responses, a fully connected SGSNN system is effectively mapped to the hardware is with a set of disconnected subtrees of radius 1 . The label propagation algorithm is not altered, but the spiking output is fundamentally affected by reducing the complexity of the spiking responses that can be generated. For the benchmark graphs studied in this article (and comparing to the results in Hamilton and Humble (2018)), we have shown that the Hamming metric remains linearly separable under appropriate binary decoding parameters and for densely connected graphs. We present these results as preliminary studies showing that spiking neuron networks can be incorporated into algorithms for graphical optimization problems.

This work has constructed a spiking implementation of community detection for deployment on neuromoprhic hardware using a set of homogeneous subtree embeddings with static parameters. Focusing on proof-of-concept, we have chosen to deploy our method on modest sized networks of 128 vertices. The close relationship between Hopfield networks, label propagation, and general q-state Potts models has led to the development of spin-glass based community detection methods with heterogeneous weighted edge sets (Ronhovde and Nussinov 2009, 2010), which have been deployed on networks with up to $10^{6}$ vertices. Future work will focus on introducing heterogeneity into the general SGSNN model, and on embedding larger subgraphs onto TrueNorth to improve the resolution of spiking label propagation.

\section{ACKNOWLEDGMENTS}

The authors thank the research group of Dr. Brian van Essen at Lawrence Livermore National Laboratory for their help and allowing us access to their IBM TrueNorth Synaptic Processor. This work was supported by the United States Department of Defense and used resources of the Computational Research and Development Programs at Oak Ridge National Laboratory. Research sponsored in part by the Laboratory Directed Research and Development Program of Oak Ridge National Laboratory, managed by UT-Battelle, LLC, for the U.S. Department of Energy.

\section{REFERENCES}

Andrew S. Cassidy, Paul Merolla, John V. Arthur, Steve K. Esser, Bryan Jackson, Rodrigo Alvarez-Icaza, Pallab Datta, Jun Sawada, Theodore M. Wong, Vitaly Feldman, et al. 2013. Cognitive computing building block: A versatile and efficient digital neuron model for neurosynaptic cores. In Proceedings of the 2013 International foint Conference on Neural Networks (IFCNN'13). IEEE, 1-10. 
Srinjoy Das, Bruno Umbria Pedroni, Paul Merolla, John Arthur, Andrew S. Cassidy, Bryan L. Jackson, Dharmendra Modha, Gert Cauwenberghs, and Ken Kreutz-Delgado. 2015. Gibbs sampling with low-power spiking digital neurons. In Proceedings of the 2015 IEEE International Symposium on Circuits and Systems (ISCAS). IEEE, 2704-2707.

Steve K. Esser, Rathinakumar Appuswamy, Paul Merolla, John V. Arthur, and Dharmendra S. Modha. 2015. Backpropagation for energy-efficient neuromorphic computing. In Advances in Neural Information Processing Systems. 1117-1125.

Steven K. Esser, Paul A. Merolla, John V. Arthur, Andrew S. Cassidy, Rathinakumar Appuswamy, Alexander Andreopoulos, David J. Berg, Jeffrey L. McKinstry, Timothy Melano, Davis R. Barch, et al. 2016. Convolutional networks for fast, energyefficient neuromorphic computing. Proc. Natl. Acad. Sci. U.S.A. 113, 41 (2016), 11441-11446.

Dan Goodman and Romain Brette. 2008. Brian: A simulator for spiking neural networks in python. Front. Neuroinform. 2 (2008), 5 pages.

Kathleen E. Hamilton, Neena Imam, and Travis S. Humble. 2017. Community detection with spiking neural networks for neuromorphic hadware. In Proceedings of the Neuromorphic Computing Symposium. ACM.

Kathleen E. Hamilton and Travis S. Humble. 2018. Spiking spin-glass models for label propagation and community detection. arXiv Preprint arXiv:1801.03571 (2018).

John Hertz, Anders Krogh, and Richard G. Palmer. 1991. Introduction to the Theory of Neural Computation. Santa Fe Institute Studies in the Sciences of Complexity, Vol. 1. Addison-Wesley.

John J. Hopfield. 1982. Neural networks and physical systems with emergent collective computational abilities. Proc. Natl Acad. Sci. U.S.A. 79, 8 (1982), 2554-2558.

John J. Hopfield and David W. Tank. 1985. Neural computation of decisions in optimization problems. Biol. Cybernet. 52, 3 (1985), 141-152.

Mark D. Humphries. 2011. Spike-train communities: Finding groups of similar spike trains. F. Neurosci. 31, 6 (2011), 2321-2336.

S. Lowel and W. Singer. 1992. Selection of intrinsic horizontal connections in the visual cortex by correlated neuronal activity. Science 255, 5041 (1992), 209-212. DOI : https://doi.org/10.1126/science.1372754 arXiv:http://science.sciencemag. org/content/255/5041/209.full.pdf

Marina Meilă. 2007. Comparing clusterings - An information based distance. f. Multivar. Anal. 98, 5 (2007), 873-895.

Paul A. Merolla, John V. Arthur, Rodrigo Alvarez-Icaza, Andrew S. Cassidy, Jun Sawada, Filipp Akopyan, Bryan L. Jackson, Nabil Imam, Chen Guo, Yutaka Nakamura, et al. 2014. A million spiking-neuron integrated circuit with a scalable communication network and interface. Science 345, 6197 (2014), 668-673.

Bruno U. Pedroni, Srinjoy Das, John V. Arthur, Paul A. Merolla, Bryan L. Jackson, Dharmendra S. Modha, Kenneth KreutzDelgado, and Gert Cauwenberghs. 2016. Mapping generative models onto a network of digital spiking neurons. IEEE Trans. Biomed. Circ. Syst. 10, 4 (2016), 837-854.

Usha Nandini Raghavan, Réka Albert, and Soundar Kumara. 2007. Near linear time algorithm to detect community structures in large-scale networks. Phys. Rev. E 76, 3 (2007), 036106.

Peter Ronhovde and Zohar Nussinov. 2009. Multiresolution community detection for megascale networks by informationbased replica correlations. Phys. Rev. E 80 (Jul. 2009), 016109. Issue 1. DOI : https://doi.org/10.1103/PhysRevE.80.016109

Peter Ronhovde and Zohar Nussinov. 2010. Local resolution-limit-free Potts model for community detection. Phys. Rev. E 81 (Apr. 2010), 046114. Issue 4. DOI : https://doi.org/10.1103/PhysRevE.81.046114

Received January 2018; accepted May 2018 